\title{
Some remarks on the elliptic Harnack inequality
}

\author{
Martin T. Barlow ${ }^{1}$ \\ Department of Mathematics \\ University of British Columbia \\ Vancouver, V6T 1 Z2 \\ Canada
}

\begin{abstract}
In this note we give three short results concerning the elliptic Harnack inequality (EHI), in the context of random walks on graphs. The first is that the EHI implies polynomial growth of the number of points in balls, and the second that the EHI is equivalent to an annulus type Harnack inequality for Green's functions. The third result uses the lamplighter group to give a counterexample concerning the relation of coupling with the EHI.
\end{abstract}

Keywords: Graph, random walk, elliptic Harnack inequality, coupling, lamplighter group.

The study of the relation between geometric properties of spaces (in particular, manifolds), and the properties of heat kernels and harmonic functions on these spaces has a long history, and continues to be very active. In particular, the papers [FS], $[\mathrm{Gg}],[\mathrm{SC}]$ establish the equivalence between the parabolic Harnack inequality (PHI), Gaussian type estimates on the heat kernel, and the condition that the underlying space satisfies volume doubling and a family of weak Poincaré inequalities. These results were translated into the graph context in [D1].

This characterizes PHI, and imply that it is stable under rough isometries. No similar characterization is known for the weaker elliptic Harnack inequality (EHI). It is trivial that PHI implies EHI, while in [BB1] and [D2] examples are given of spaces which satisfy EHI but fail to satisfy the PHI. The example in [D2] shows in addition that EHI does not imply volume doubling. See $[\mathrm{HSC}]$ for some further results on the difference between EHI and PHI.

In this note we give three short results concerning the EHI. For simplicity we give these in the easiest context, that of random walks on graphs. The first is that EHI implies polynomial growth of the number of points in balls, and the second that EHI is equivalent to an annulus type Harnack inequality for Green's functions. The final result uses the lamplighter group to show that a coupling type condition for random walks does not imply EHI. The paper is concluded with an open problem.

We begin by recalling the definition of a weighted graph. Let $\Gamma=(G, E)$ be an locally finite connected graph. If $x$ and $y$ are connected by an edge $\{x, y\}$ we write $x \sim y$. We call $\nu=\left(\nu_{x y}\right), x, y \in G$ a conductance matrix if $\nu_{x y} \geq 0, \nu_{x y}=\nu_{y x}$ for all $x, y \in G$ and in addition $\nu$ is linked to the graph structure by the condition that $\nu_{x y}>0$ if and only if

1 Research partially supported by grants from NSERC (Canada) and CNRS (France). 
$x \sim y$. The triple $(G, E, \nu)$ is called a weighted graph. We call the natural weight on $\Gamma$ the weights given by taking $\nu$ to be the adjacency matrix of $\Gamma$; that is $\nu_{x y}=1$ if and only if $x \sim y$. Let $\mu(x)=\sum_{y} \nu_{x y}$; we extend $\mu$ to a measure on $G$.

Given a weighted graph $(\Gamma, \nu)$ we define the simple random walk $X$ on $G$ to be the Markov chain with transition probabilities given by

$$
p_{x y}=\mathbb{P}^{\cdot}\left(X_{n+1}=y \mid X_{n}=x\right)=\frac{\nu_{x y}}{\mu(x)}, \quad x, y \in G, n \geq 0 .
$$

Let $d(x, y)$ be the graph distance on $\Gamma$. For $x \in G, r \in(0, \infty)$, let

$$
B(x, r)=\{y: d(x, y) \leq r\} .
$$

Note that any pair of points $x$ and $y$ will be connected by a geodesic path of length $n=d(x, y)$, but that this path will not in general be unique. We select a family $\gamma(x, y)$, $x, y \in G$ of geodesic paths.

Definition. 1. Let $A \subset G$. We write $\partial A=\left\{y \in A^{c}: d(x, y)=1\right.$ for some $\left.x \in A\right\}$ for the exterior boundary of $A$, and set $\bar{A}=A \cup \partial A$, and write $\bar{B}(x, r)=B(x, r) \cup \partial B(x, r)$.

2. Define the Laplacian on $(\Gamma, \nu)$ by

$$
\Delta f(x)=\frac{1}{\mu_{x}} \sum_{y} \nu_{x y}(f(y)-f(x)) .
$$

3. A function $h$ is harmonic on $A \subset G$ if $h: \bar{A} \rightarrow \mathbb{R}$ and $\Delta h(x)=0, x \in A$.

We now introduce two conditions that a weighted graph $(\Gamma, \nu)$ may or may not satisfy.

Definition. 1. $(\Gamma, \nu)$ has controlled weights if there exists $p_{0}>0$ such that for all $x \in G$

$$
\frac{\nu_{x y}}{\mu(x)} \geq p_{0} .
$$

(This was called the $p_{0}$-condition in [GT2].)

2. $(\Gamma, \nu)$ satisfies an elliptic Harnack inequality (EHI) if there exists $C_{1}>0$ such that, for any $x \in G, R \geq 1$, and non-negative $h: G \rightarrow \mathbb{R}$ harmonic in $B(x, 2 R)$,

$$
\sup _{B(x, R)} h \leq C_{1} \inf _{B(x, R)} h .
$$

We have taken balls $B(x, R) \subset B(x, 2 R)$ just for simplicity. If $(\Gamma, \nu)$ has controlled weights, $K>1$ and (3) holds whenever $h \geq 0$ is harmonic in $B(x, K R)$, then an easy chaining argument gives (EHI) (for a different constant $C_{1}$ ).

Note that if $(\Gamma, \nu)$ has controlled weights then it satisfies a local Harnack inequality: if $h \geq 0$ is harmonic on $A \subset G$ then

$$
h(x) \geq p_{0} h(y), \quad \text { if } x \in A, \quad y \sim x .
$$

It follows immediately that any finite graph satisfies (EHI), but it may still be of interest to ask how good the constant $C_{1}$ can be. 
Remark. A hypothesis such as controlled weights is needed to connect the graph structure with the Laplacian. One might hope that (EHI) would imply controlled weights, but this is not the case. Consider the graph $\Gamma$ with $G=\mathbb{Z} \times\{0,1,2\}$, and edges

$$
E=\{\{(n, 0),(n+1,0)\},\{(n, i),(n, j)\}, n \in \mathbb{Z}, i, j=0,1,2, i \neq j\} .
$$

Let $\nu_{(n, 1),(n, 2)}=2^{-|n|}, n \in \mathbb{Z}$, and all other edges have weight 1 . Then $(\Gamma, \nu)$ does not have controlled weights but it is easy to verify that (EHI) holds.

The first main result of this paper is that (EHI) implies a bound on the size of the balls $B(x, R)$. Write $|A|$ for the number of elements in the set $A$.

Theorem 1. Let $(G, E, a)$ be a weighted graph with controlled weights which satisfies (EHI) with a constant $C_{1}$. Then there exist constants $C, \theta$, depending only on $C_{1}$ such that

$$
\left|B\left(x_{0}, R\right)\right| \leq C R^{1+\theta}, \quad x_{0} \in G, R \geq 1 .
$$

Proof. Let $B=B\left(x_{0}, R\right), \tau=\min \left\{n: X_{n} \in \partial B\right\}$. Fix $z \in \partial B$, and let

$$
h(x)=h_{z}(x)=\mathbb{P}^{x}\left(X_{\tau}=z\right) .
$$

Let $y_{i}$ be a sequence of points on the geodesic path $\gamma\left(z, x_{0}\right)$ with $d\left(y_{j}, z\right)=3^{j}$, for $0 \leq j \leq N$, where $N$ is chosen so that $3^{N} \leq R<3^{N+1}$. Choose also $z_{j}$ on this geodesic with $d\left(z, z_{j}\right)=2.3^{j}$, for $0 \leq j \leq N$, except that if $2.3^{N}>R$ then we take $z_{N}=x_{0}$. Applying (EHI) to $h$ in $B\left(z_{j}, 3^{j}\right) \subset B\left(z_{j}, 2.3^{j}\right)$ we have, if $0 \leq j<N$

$$
h\left(y_{j+1}\right) \geq C_{1}^{-1} h\left(y_{j}\right),
$$

while if $j=N$ we obtain $h\left(x_{0}\right) \geq C_{1}^{-1} h\left(y_{N}\right)$.

The local HI implies that $h\left(y_{0}\right) \geq p_{0} h(z)=p_{0}$, and therefore

$$
h_{z}\left(x_{0}\right) \geq \frac{p_{0}}{C_{1}} C_{1}^{-N} \geq \frac{p_{0}}{C_{1}} R^{-\theta},
$$

where $\theta=\log C_{1} / \log 3$. Since

$$
1=\sum_{z} h_{z}\left(x_{0}\right) \geq|\partial B| c_{1} R^{-\theta}
$$

we obtain $\left|\partial B\left(x_{0}, R\right)\right| \leq c R^{\theta}$, and summing over $R$ gives (4).

Remarks. 1. This proof controls the number of vertices in $B\left(x_{0}, R\right)$ rather than $V\left(x_{0}, R\right)=\mu\left(B\left(x_{0}, R\right)\right)$; I do not know if one has similar control of $V\left(x_{0}, R\right)$.

2. Note that if $\left|B\left(x_{0}, R\right)\right| \geq R^{1+\delta}$ then this proof implies that $C_{1} \geq 3^{\delta}$. 
We now recall the definition of the Green's function on $(\Gamma, \nu)$. Let $D \subset G, \tau_{D}=\min \{n \geq$ $\left.0: X_{n} \notin D\right\}$, and $p_{n}^{D}(x, y)=\mathbb{P}^{x}\left(X_{n}=y, n<\tau_{D}\right) / \mu(y)$. Then we set

$$
g_{D}(x, y)=\sum_{n=0}^{\infty} p_{n}^{D}(x, y) .
$$

Sufficient conditions for $g_{D}$ to be finite are that $D$ is finite, or that $(\Gamma, \nu)$ is transient. $g_{D}$ is symmetric in $x, y$, is zero if either variable is outside $D$, and $g_{D}\left(x_{0}, \cdot\right)$ is harmonic in $D-\left\{x_{0}\right\}$.

In [GT2] the following condition (HG) is introduced.

Definition. $(\Gamma, \nu)$ satisfies (HG) if there exists a constant $C_{2} \geq 1$ such that, for any finite set $D \subset G$, if $R \geq 1$ and $B\left(x_{0}, 2 R\right) \subset D$ then

$$
\max _{y \in B\left(x_{0}, R\right)^{c}} g_{D}\left(x_{0}, y\right) \leq C_{2} \min _{y \in B\left(x_{0}, R\right)} g_{D}\left(x_{0}, y\right) .
$$

In [GT1] and [GT2] it is proved that (HG) implies (EHI), and that (EHI) and another (geometric) condition, denoted (BC), implies (HG). The point of the next result is that no additional geometric condition is needed.

Theorem 2. Suppose $(\Gamma, \nu)$ has controlled weights and satisfies (EHI). Then there exists a constant $C_{2}$ such that if $x_{0} \in G, R \geq 1, d\left(x_{0}, x\right)=d\left(x_{0}, y\right)=R$, and $B\left(x_{0}, 2 R\right) \subset D$ then

$$
C_{3}^{-1} g_{D}\left(x_{0}, y\right) \leq g_{D}\left(x_{0}, x\right) \leq C_{3} g_{D}\left(x_{0}, y\right) .
$$

In particular the conditions (EHI) and (HG) are equivalent.

Proof. Note first that by symmetry it is enough to prove the right hand inequality. Using the local Harnack inequality we can strengthen (EHI) to give, if $d\left(x_{0}, x\right)=r$, that

$$
\max _{B(x, r / 2)} g_{D}\left(x_{0}, .\right) \leq C_{4} \min _{B(x, r / 2)} g_{D}\left(x_{0}, .\right) .
$$

We first assume that $R \geq 12$ and is divisible by 12 . Let $x^{\prime}, y^{\prime}$ be the midpoints of $\gamma\left(x_{0}, x\right)$, and $\gamma\left(x_{0}, y\right)$. Thus $d\left(x_{0}, x^{\prime}\right)=d\left(x_{0}, y^{\prime}\right)=R / 2$. Clearly we have $d\left(x^{\prime}, y\right) \geq R / 2$ and $d\left(x, y^{\prime}\right) \geq R / 2$.

We now consider two cases.

Case 1. $d\left(x^{\prime}, y^{\prime}\right) \leq R / 3$. Let $z$ be as close as possible to the midpoint of $\gamma\left(x^{\prime}, y^{\prime}\right)$. Then $d\left(z, x^{\prime}\right) \leq 1+R / 6 \leq R / 4$. So applying (EHI) to $g_{D}\left(x_{0}, \cdot\right)$ in $B\left(x^{\prime}, R / 4\right) \subset B\left(x^{\prime}, R / 2\right)$, we deduce that

$$
C_{4}^{-1} g_{D}\left(x_{0}, x^{\prime}\right) \leq g_{D}\left(x_{0}, z\right) \leq C_{4} g_{D}\left(x_{0}, x^{\prime}\right) .
$$

Now apply (EHI) to $g_{D}\left(x_{0}, \cdot\right)$ in $B(x, R / 2) \subset B(x, R)$, to deduce that

$$
C_{4}^{-1} g_{D}\left(x_{0}, x\right) \leq g_{D}\left(x_{0}, x^{\prime}\right) \leq C_{4} g_{D}\left(x_{0}, x\right) .
$$


Combining these inequalities we deduce that

$$
C_{4}^{-2} g_{D}\left(x_{0}, x\right) \leq g_{D}\left(x_{0}, z\right) \leq C_{4}^{2} g_{D}\left(x_{0}, x\right),
$$

and this, with a similar inequality for $g_{D}\left(x_{0}, y\right)$, proves $(5)$.

Case 2. $d\left(x^{\prime}, y^{\prime}\right)>R / 3$. Apply (EHI) to $g_{D}(y, \cdot)$ in $B\left(x_{0}, R / 2\right) \subset B\left(x_{0}, R\right)$, to deduce that

$$
C_{4}^{-1} g_{D}\left(y, x^{\prime}\right) \leq g_{D}\left(y, x_{0}\right) \leq C_{4} g_{D}\left(y, x^{\prime}\right) .
$$

Now look at $g_{D}\left(x^{\prime}, \cdot\right)$. If $z^{\prime}$ is on $\gamma\left(y^{\prime}, y\right)$ with $d\left(y^{\prime}, z^{\prime}\right)=s \in[0, R / 2]$ then as $d\left(x^{\prime}, y^{\prime}\right)>R / 3$ and $d\left(x^{\prime}, y\right) \geq R / 2$ we have $d\left(x^{\prime}, z^{\prime}\right) \geq \max (R / 3-s, s)$. Hence we deduce $d\left(x^{\prime}, z^{\prime}\right) \geq R / 6$. So applying (EHI) repeatedly to $g_{D}\left(x^{\prime}, \cdot\right)$ for a chain of balls $B\left(z^{\prime}, R / 12\right) \subset B\left(z^{\prime}, R / 6\right)$ we deduce that

$$
C_{4}^{-6} g_{D}\left(x^{\prime}, y^{\prime}\right) \leq g_{D}\left(x^{\prime}, y\right) \leq C_{4}^{6} g_{D}\left(x^{\prime}, y^{\prime}\right) .
$$

So, we obtain from (7) and (8),

$$
g_{D}\left(y, x_{0}\right) \leq C_{4} g_{D}\left(y, x^{\prime}\right) \leq C_{4}^{7} g_{D}\left(x^{\prime}, y^{\prime}\right), \quad g_{D}\left(x^{\prime}, y^{\prime}\right) \leq C_{4}^{6} g_{D}\left(y, x^{\prime}\right) \leq C_{4}^{7} g_{D}\left(y, x_{0}\right) .
$$

We have similar inequalities relating $g_{D}\left(x, x_{0}\right)$ and $g_{D}\left(x^{\prime}, y^{\prime}\right)$, which proves (5).

If $R \leq 11$ then (5) follows using the local Harnack inequality. If $R \geq 12$ is not divisible by 12 , let $R^{\prime}=12 k$ with $R-11 \leq R^{\prime}<R$, and choose points $x_{1}$ and $y_{1}$ a distance $R^{\prime}$ from $x_{0}$ with $d\left(x, x_{1}\right) \leq 11, d\left(y, y^{\prime}\right) \leq 11$. Then we have (5) for $x_{1}$ and $y_{1}$, and (5) for $x$ and $y$ again follows using the local Harnack inequality.

Finally, to deduce (HG) note that by the maximum principle the maximum and minimum in (HG) will be attained at a point $y$ with $d\left(x_{0}, y\right)=R$ and $z$ with $d\left(x_{0}, z\right)=R-1$. Hence using the local Harnack inequality (HG) follows from (5).

The final question we consider is the relation between (EHI) and coupling for random walks on $(\Gamma, \nu)$. For further details on coupling see $[\mathrm{Lv}]$. It is sometimes easier to couple continuous time random walks (CTSRW), rather than discrete time ones, so we recall that the continuous time simple random walk $Y_{t}$ on $(\Gamma, \nu)$ is the Markov process with generator $\Delta f(x) . Y$ waits for an exponential time with mean 1 at each vertex $x$, and then moves to each $y \sim x$ with the same probability as $X$, that is $p_{x y}=\nu_{x y} / \mu(x)$.

Definition. 1. Let $K>1$. ( $\Gamma, \nu)$ satisfies uniform (co-adapted) coupling with constant $K$, if for all $x_{0} \in G, R \geq 1$, and $y_{1}, y_{2} \in B\left(x_{0}, R\right)$ there exist CTSRW walks $Y_{t}^{i}$ with $Y_{0}^{i}=y_{i}$ satisfying the following condition. Let

$$
\begin{aligned}
\tau_{C} & =\min \left\{t \geq 0: Y_{t}^{1}=Y_{t}^{2}\right\}, \\
\tau_{E} & =\min \left\{t \geq 0: Y_{t}^{1} \notin B\left(x_{0}, K R\right) \text { or } Y_{t}^{2} \notin B\left(x_{0}, K R\right)\right\} .
\end{aligned}
$$

Then there exists $p_{1}>0$, independent of $x_{0}$ and $R$, such that $\mathbb{P}\left(\tau_{C}<\tau_{E}\right)>p_{1}$. We denote this condition $\mathrm{UC}(K)$.

2. Write $\operatorname{Osc}(h, A)=\max _{A} h-\min _{A} h .(\Gamma, \nu)$ satisfies an oscillation inequality with 
constant $K>1$ (denoted $(\mathrm{OI}(K))$, if there exists $\rho<1$ such that, if $h$ is harmonic in $B(x, K R)$ then

$$
\operatorname{Osc}(h, B(x, R)) \leq \rho \operatorname{Osc}(h, \bar{B}(x, K R)) .
$$

Remarks. 1. In the above we require that the $Y^{i}$ are defined on the same filtered probability space.

2. Some of the consequences of EHI, such as the Liouville property for bounded harmonic functions on $\Gamma$, also follow from $\mathrm{OI}(K)$.

3. There are several different types of coupling - see $[\mathrm{CG}]$ for a weaker type.

It is easy to see that uniform coupling implies $\mathrm{OI}(K)$. For, if $h$ is harmonic on $B\left(x_{0}, K R\right)$ and we write $\operatorname{Osc}(h, A)=\max _{a} h-\min _{A} h$ then, writing $B=B\left(x_{0}, R\right), B^{*}=$ $\bar{B}\left(x_{0}, K R\right), T=\tau_{C} \wedge \tau_{E}$, and choosing $x_{i}$ suitably,

$$
\begin{aligned}
\operatorname{Osc}(h, B) & =h\left(x_{2}\right)-h\left(x_{1}\right) \\
& =\mathbb{E}\left(h\left(Y_{T}^{2}\right)-h\left(Y_{T}^{1}\right)\right) \\
& \leq \mathbb{P}\left(\tau_{E}<\tau_{C}\right) \operatorname{Osc}\left(h, B^{*}\right) \leq\left(1-p_{1}\right) \operatorname{Osc}\left(h, B^{*}\right) .
\end{aligned}
$$

It is also well known that EHI implies $\mathrm{OI}(K)$, and that $\mathrm{OI}(K)$ plus a suitable lower bound on the hitting probabilities of small balls implies EHI. (See for example [FS] or [BB1]). The following example on the lamplighter group shows that this extra condition is needed: uniform coupling alone is not enough to imply EHI. It is also shows that (unlike $\mathrm{EHI})$ the size of the larger ball $B(x, K R)$ plays an important role in the conditions $\mathrm{UC}(K)$ and $\mathrm{OI}(K)$.

For details of the lamplighter group see for example [LPP] or [W]. Let

$$
\mathcal{A}=\left\{\xi \in\{0,1\}^{\mathbb{Z}}: \sum_{k} \xi_{k}<\infty\right\}
$$

and $G=\mathbb{Z} \times \mathcal{A}$. We denote points $x \in G$ by $x=(n, \xi)$, and will write $A(x)=A(n, \xi)=$ $\left\{i: \xi_{i}=1\right\}$. For $\xi \in \mathcal{A}, k=0,1$ let $T_{n, k}(\xi) \in\{0,1\}^{\mathbb{Z}}$ be the sequence $\eta$ with $\eta_{i}=\xi_{i}, i \neq n$, $\eta_{n}=k$. We define edges on $G$ by taking the four neighbours of $(n, \xi)$ to be the points ( $\left.n \pm 1, T_{n, k}(\xi)\right), k=0,1$. One thinks of the $\xi_{i}$ as lamps (off or on), and $n$ as the location of the lamplighter. Each move on the graph, the lamplighter may switch the status of the current lamp, and then moves to a neighbouring point. We write $Y_{t}=\left(U_{t}, \Xi(t)\right)$ for the CTSRW on $\Gamma$. Note that $U$ is a CTSRW on $\mathbb{Z}$ and that after $U$ leaves any site $n$ the state of the lamp $\Xi_{n}$ has been randomized. (This is the 'switch then walk' random walk on $G$; there are several other natural random walks, such as 'walk then switch' or 'switch, walk, switch' - see [W] for more details).

Theorem 3. (a) The lamplighter group $G$ satisfies uniform coupling for $K>4$.

(b) $G$ fails to satisfy $\mathrm{OI}(K)$ for $K<3$, and in particular does not satisfy EHI.

Proof. (a) By the symmetry of the group we can take $x_{0}=\left(0, \eta^{0}\right)$, where $\eta_{i}^{0}=0$ for all $i$. If $x=\left(x^{\prime}, \xi\right) \in G$, with $-a=\min \left\{n: \xi_{n}=1\right\}, b=\max \left\{n: \xi_{n}=1\right\}$, and $x^{\prime} \geq 0$ then it is easy to check that $d\left(x_{0}, x\right)=2 a+b+\left|b-x^{\prime}\right|$. 
Let $x_{i}=\left(n_{i}, \xi^{i}\right) \in B\left(x_{0}, R\right), i=1,2$. Then we clearly have $\left|n_{i}\right| \leq R$ and $\left\{n: \xi_{n}^{i}=\right.$ $1\} \subset[-R, R]$. Let $0<\varepsilon<(K-4) / 8$. We now define $Y_{t}^{i}$ with initial states $x_{i}$ as follows. First, we try to couple the CTRSW $U^{1}$ and $U^{2}$ using reflection coupling - see [LR]. With probability $p_{1}=p_{1}(\varepsilon)>0$, independent of $R$, this coupling succeeds at a time $T_{1}$ before either $U^{1}$ or $U^{2}$ has left $I(\varepsilon)=[-R(1+\varepsilon), R(1+\varepsilon)]$. Denote this event $F_{1}$.

On $F_{1}$ we have $\left\{n: \Xi_{n}^{i}\left(T_{1}\right)=1\right\} \subset I(\varepsilon)$. If $F_{1}$ occurs, we now continue by taking $U_{t}=U_{t}^{1}=U_{t}^{2}$ for $t \geq T_{1}$, and by making each lamp randomization the same for the two processes. Hence $\Xi_{n}^{i}(t)$ agree for every site $n$ visited by $U$ between $T_{1}$ and $t$.

Let $F_{2}$ be the event that $U$ hits both $\pm R(1+\varepsilon)$ before leaving $I(2 \varepsilon)$; then $\mathbb{P}\left(F_{2} \mid F_{1}\right)>$ $p_{2}>0$. Let $T_{2}$ be the exit time from $I(2 \varepsilon)$. Then $\mathbb{P}\left(F_{1} \cap F_{2}\right) \geq p_{1} p_{2}$ and on $F_{1} \cap F_{2}$ we have $Y_{T_{2}}^{1}=Y_{T_{2}}^{2}$, and $\left\{n: \Xi_{n}^{i}(t)=1\right\} \subset I(2 \varepsilon), U_{t}^{i} \in I(2 \varepsilon)$, for each $t \in\left[0, T_{2}\right]$. Thus on $F_{1} \cap F_{2} d\left(x_{0}, Y_{t}^{i}\right) \leq 4(1+2 \varepsilon) R<K R$ for $t \in\left[0, T_{2}\right]$, so that $T_{2}<\tau_{E}$. This proves that $\Gamma$ satisfies uniform coupling with constant $K$.

(b) We now prove that OI with $K<3$ fails for $G$. For this calculation it is easier to use the discrete time random walk $X_{n}=\left(V_{n}, \Theta(n)\right)$; of course $X$ and $Y$ have the same harmonic functions. Note that if $T$ is a stopping time with respect to $V$, then at time $T$ the states of all the lamps $\Theta_{n}(T), n \in\left\{V_{k}, 0 \leq k \leq T-1\right\}$ will have been randomized.

Let $\delta=(3-K) / 3, \lambda=1-\delta$. Let $\tau=\min \left\{n: d\left(x_{0}, X_{n}\right)>K R\right\}$, set $G=$ $\left\{A\left(X_{\tau}\right) \cap[\lambda R, R] \neq \emptyset\right\}$ and let

$$
h(y)=\mathbb{P}^{y}(G), \quad y \in B\left(x_{0}, K R\right) .
$$

Let $y_{1}=\left(-R, \xi^{(1)}\right), y_{2}=\left(R, \xi^{(2)}\right)$, where $\xi_{n}^{(1)}=1_{[-R, 0]}(n)$ and $\xi_{n}^{(2)}=1_{[0, R]}(n)$.

We begin by bounding $h\left(y_{1}\right)$. Let $V_{0}=-R$, and $T=\min \left\{n: V_{n} \notin(-3 R, \lambda R)\right\}$. Note that since $A\left(X_{T}\right) \cap[\lambda R, R]=A\left(X_{0}\right) \cap[\lambda R, R]$, we have $G \subset\{T<\tau\}$. So

$$
h\left(y_{1}\right)=\mathbb{P}^{y_{1}}(G \cap\{T<\tau\}) \leq \mathbb{P}^{y_{1}}(T<\tau) .
$$

Let $-Z=\min \left\{n: \Theta_{n}(T)=1\right\}$. If $T<\tau$ then $V_{T}=\lambda R$, and we have

$$
d\left(x_{0}, X_{T}\right)=2 Z+V_{T}=2 Z+\lambda R<K R,
$$

so that $Z \leq \lambda R$. Thus at time $T$ every lamp in $[-R,-\lambda R)$ must have been switched off, and we deduce that

$$
\mathbb{P}^{y_{1}}(G) \leq 2^{-\delta R} .
$$

Similar considerations lead one to expect that $\mathbb{P}^{y_{2}}\left(G^{c}\right) \leq c 2^{-\delta R}$, but the argument is a little more involved. A cruder bound is easier to obtain, and enough here. Let $V_{0}=R$, and note that, for any $n \geq 0$,

$$
\mathbb{P}^{y_{2}}\left(A\left(X_{n}\right) \cap(\lambda R, R]=\emptyset\right)=2^{-\delta R} .
$$

Let $T$ be the first hit by $V$ on $\pm 3 R$ : we have $T>\tau$. So

$$
\begin{aligned}
\mathbb{P}^{y_{2}}\left(G^{c}\right) & \leq \mathbb{P}^{y_{2}}\left(T \geq R^{3}\right)+\mathbb{P}^{y_{2}}\left(G^{c} ; V<R^{3}\right) \\
& \leq c R^{-1}+\mathbb{P}^{y_{2}}\left(A\left(X_{n}\right) \cap(\lambda R, R]=\emptyset \text { for some } 0 \leq n<R^{3}\right) \leq c R^{-1}+R^{3} e^{-\delta R} .
\end{aligned}
$$

So $\operatorname{Osc}\left(h, \bar{B}\left(x_{0}, R\right)\right) \geq h\left(y_{2}\right)-h\left(y_{1}\right)=1-o(R)$, and hence $\operatorname{OI}(K)$ fails for $G$.

Finally, since EHI implies OI for any $K>1$, EHI also fails for $G$. 
Remarks. 1. [W] identifies the cone of positive harmonic functions associated with the 'walk then switch' and the 'switch, walk, switch' walks on $G$ : in both cases there are nonconstant positive harmonic functions.

2. The key feature used by the argument in Theorem 3 is that after $U$ has moved across an interval $I \subset \mathbb{Z}$ all the lamps in $I$ will have been randomized. So this proof also works for the 'walk then switch' and 'switch, walk, switch' walks. But it does not apply to the 'walk or switch' walk.

3. It seems likely that there is a gap between the best constant $K$ for $\mathrm{UC}(K)$ and $\mathrm{OI}(K)$.

We conclude this paper by mentioning an open problem. If $D \subset G$ we define for $f: D \rightarrow \mathbb{R}$ the Dirichlet form on $L^{2}(D)$

$$
\mathcal{E}_{D}(f, f)=\sum_{x \in D} \sum_{y \in D} \nu_{x y}(f(x)-f(y))^{2} .
$$

For disjoint $A, B \subset D$ define the conductance

$$
C_{D}(A, B)=\inf \left\{\mathcal{E}_{D}(f, f): f=1 \text { on } A \text { and } f=0 \text { on } B\right\} .
$$

Given $x_{0} \in G, R \geq 10$ let

$$
\mathcal{D}\left(x_{0}, R\right)=\left\{(x, y): d\left(x, x_{0}\right) \leq R / 2, d\left(y, x_{0}\right) \leq R / 2, d(x, y) \geq R / 3\right\}
$$

We introduce the following condition.

Definition. $(\Gamma, \nu)$ satisfies the dumbbell condition (DB) if there exists a constant $C_{3}<\infty$ such that for all $x_{0} \in G, R \geq 10$, writing $D=B\left(x_{0}, R\right), \mathcal{D}=\mathcal{D}\left(x_{0}, R\right)$,

$$
\max _{(x, y) \in \mathcal{D}} C_{D}(B(x, R / 10), B(y, R / 10)) \leq C_{3} \min _{(x, y) \in \mathcal{D}} C_{D}(B(x, R / 10), B(y, R / 10)) .
$$

Note that $(\mathrm{DB})$ is stable under bounded perturbation of the weights $\nu_{x y}$ : if $(\Gamma, \nu)$ satisfies (DB) and $\nu_{x y}^{\prime}$ satisfy, for some constant $c_{1}$,

$$
c_{1}^{-1} \nu_{x y} \leq \nu_{x y}^{\prime} \leq c_{1} \nu_{x y}, \quad x, y \in G
$$

then $\left(\Gamma, \nu^{\prime}\right)$ also satisfies $(\mathrm{DB})$.

Problem. Suppose $(\Gamma, \nu)$ has controlled weights. Is (DB) equivalent to EHI?

Acknowledgment. I am grateful for Terry Lyons for some stimulating conversations related to this paper.

\section{References}

[BB1] M.T. Barlow and R.F. Bass, Brownian motion and harmonic analysis on Sierpinski carpets. Canad. J. Math. 51 (1999) 673-744.

[CG] M. Cranston, A. Greven. Coupling and harmonic functions in the case of continuous time markov processes. Stoch. Proc. Appl. 60 (1995), 261-286. 
[D1] T. Delmotte. Parabolic Harnack inequality and estimates of Markov chains on graphs. Rev. Math. Iberoamericana 15 (1999), 181-232.

[D2] T. Delmotte. Graphs between the elliptic and parabolic Harnack inequalities. Potential Anal. 16 (2002), 151-168.

[FS] E.B. Fabes and D.W. Stroock. A new proof of Moser's parabolic Harnack inequality via the old ideas of Nash. Arch. Mech. Rat. Anal. 96 (1986) 327-338.

[Gg] A.A. Grigor'yan. The heat equation on noncompact Riemannian manifolds. Math. USSR Sbornik 72 (1992) 47-77.

[GT1] A. Grigor'yan, A. Telcs. Sub-Gaussian estimates of heat kernels on infinite graphs. Duke Math. J. 109 (2001) 452-510.

[GT2] A. Grigor'yan, A. Telcs. Harnack inequalities and sub-Gaussian estimates for random walks. Math. Annalen 324 (2002), 521-556.

[HSC] W. Hebisch, L. Saloff-Coste. On the relation between elliptic and parabolic Harnack inequalities. Ann. Inst. Fourier (Grenoble) 51 (2001), 1437-1481.

[Lv] T. Lindvall, Lectures on the coupling method. Wiley, New York, 1992.

[LR] T. Lindvall and L.C.G. Rogers. Coupling of multi-dimensional diffusions by reflection. Ann. Prob. 14 (1986) 860-872.

[LPP] R. Lyons, R. Pemantle, Y. Peres. Random walks on the lamplighter group. Ann. Probab. 24 (1996), no. 4, 1993-2006.

[SC] L. Saloff-Coste. A note on Poincaré, Sobolev, and Harnack inequalities. Duke Math. J. 65 (1992), Inter. Math. Res. Notices 2 (1992) 27-38.

[W] W. Woess. Lamplighters, Diestel-Leader graphs, random walks, and harmonic functions. Preprint 2003.

Version 1.01 September 2003 